\title{
Sustainability issues in hole making technologies: current practices and challenges
}

\begin{abstract}
The recent significant increase in demand for composite materials has been associated with higher production of waste. It is estimated that composite scrap makes up between $5 \%$ and $40 \%$ of the total composite production volume. To produce holes, various methods have been proposed and developed, usually related to machining such as drilling and milling. These processes tend to produce scrap or waste after completing the hole-making process. This chapter is a comprehensive review of sustainability issues in hole-making technologies. The discussion covers three aspects that should be taken into consideration in ensuring sustainability in hole-making. The three aspects represent the phases before, during, and after the composite is prepared until the end of the material's life. Based on the literature, promoting the methods that have been developed and the benefits that can be obtained from hole-making technologies is key to successfully implementing sustainability.
\end{abstract}

Keyword: Hole-making; Composite; Sustainability; Environment 